\title{
Downregulated microRNA-133a induces HUVECs injury: Potential role of the (pro) renin receptor in angiotensin II-dependent hypertension
}

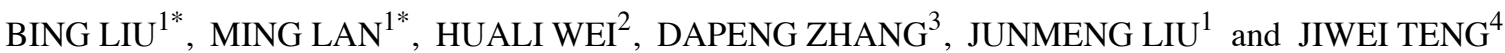 \\ ${ }^{1}$ Department of Cardiology, Beijing Hospital, National Center of Gerontology of China, Beijing 100730; \\ ${ }^{2}$ Department of Gynecology and Obstetrics, China Meitan General Hospital; \\ ${ }^{3}$ Heart Center and Beijing Key Laboratory of Hypertension Disease, Beijing Chaoyang Hospital, \\ Capital Medical University, Beijing 100028; ${ }^{4}$ Department of Cardiovascular Medicine, \\ The Second Affiliated Hospital of The Fourth Military Medical University, Xi'an, Shaanxi 710038, P.R. China
}

Received January 8, 2019; Accepted June 12, 2019

DOI: $10.3892 / \mathrm{mmr} .2019 .10519$

\begin{abstract}
The renin-angiotensin system (RAS) serves an essential role in hypertension. MicroRNAs (miRs) have been reported to be important regulators in angiotensin (Ang) II-dependent hypertension. We aimed to explore the roles of Ang II and miR-133a in the mechanism underlying hypertension. Human umbilical vein endothelial cells (HUVECs) were identified by immunofluorescence staining. Cell viability and miR-133a expression under the inhibition of Ang II of various concentrations were determined by an MTT assay and reverse transcription-quantitative polymerase chain reaction (RT-qPCR), respectively. The effects of HUVECs transfected with miR-133a mimic or inhibitor on Ang II-induced apoptosis were measured using flow cytometry. The potential targeting of miR-133a to the 3 ' untranslated region of (pro) renin receptor (PRR) was assessed using TargetScan and a dual-luciferase assay. The effects of PRR interference using small interfering (si)RNA on PRR expression and the rate of apoptosis were determined by RT-qPCR, western blotting and flow cytometry, respectively. Ang II at a concentration of $10^{-5} \mathrm{M}$ significantly inhibited the cell viability $(\mathrm{P}<0.05)$ and miR-133a expression $(\mathrm{P}<0.01)$; Downregulation of miR-133a suppressed cell viability. HUVECs transfected with miR-133a mimic reduced the rate of Ang II-induced apoptosis from 21.99
\end{abstract}

Correspondence to: Dr Jiwei Teng, Department of Cardiovascular Medicine, The Second Affiliated Hospital of The Fourth Military Medical University, 1 Xinsi Road, Baqiao, Xi'an, Shaanxi 710038, P.R. China

E-mail: jiweit_tengjw1@163.com

*Contributed equally

Key words: cardiovascular disease, human umbilical vein endothelial cells, microRNA, renin-angiotensin system, dual luciferase assay to $12.38 \%$, but miR-133a inhibitor promoted Ang II-induced apoptosis (apoptosis rate, 28.9\%). PRR was predicted to be a target gene of miR-133a. Transfection with siPRR decreased the apoptotic rate in Ang II + negative control and Ang II + miR-133a inhibitor group to 11.39 and $12.94 \%$, respectively. Our findings also suggested that Ang II promoted PRR expression to enhance the apoptotic rate of HUVECs via the suppression of miR-133a. Furthermore, siPRR efficiently decreased the Ang II-induced apoptosis.

\section{Introduction}

Hypertension is one of the most common chronic diseases that is accompanied with a variety of severe complications, including kidney failure, myocardial infarction and atherosclerosis (1). Structural alterations or vascular remodeling, increased stiffness and endothelial dysfunction are key features of hypertension $(2,3)$. Vascular endothelial cells form a layer of flat squamous cells located in the lining of the blood vessels and, serve roles in maintaining the integrity and permeability of the vessel wall and regulating vascular tone (4). Once the vascular endothelial cells experience damage, the synthesis and secretion angiotensin (Ang) II is promoted, leading to vasoconstriction and increases in blood pressure $(5,6)$. Ang II was reported to be the most important element in the renin-angiotensin system (RAS) (7). Several clinical applications revealed that blocking the angiotensin II receptor could not only suppress variations in and reduce blood pressure, but also decreased the morbidity and mortality associated with cardiovascular diseases $(8,9)$.

Recently, the (pro) renin receptor (PRR) has received widespread attention as a novel component of the RAS. It was reported that both the knockdown and overexpression of PRR gene in local tissues had notable effects on local-tissue RAS and blood pressure regulation (10,11). Increased expression of PRR has been detected in the nephritic collecting duct of Ang II-induced rat models and the plasma of patients with hypertension $(12,13)$. PRR knockout could significantly suppress Ang II-induced hypertension and the production of 
Ang II in the brain of human renin-angiotensinogen double transgenic mice (14). Interestingly, high-expression PRR also has a promoting effect on Ang II production, suggesting an augmentation in positive feedback between PRR and Ang II $(15,16)$.

MicroRNA (miRNA/miR) is a single-strand RNA molecule of 21-24 nucleotides, which plays a vital role in the development and homeostasis of tissues and organs by inhibiting protein translation at the posttranscriptional level, or promoting mRNA degradation (17). Previous studies indicated that the marked difference in the miRNA expression profile in the peripheral blood of patients with hypertension contributed to the early diagnosis and the prognosis of complications of this condition $(18,19)$. At present, investigations with different experimental models have revealed that miR-133a protected against myocardial fibrosis and modulated electrical repolarization $(20,21)$. In vivo experiments demonstrated that Ang II caused an increase in systolic blood pressure and myocardial fibrosis in under conditions of downregulated miR-133a and miR-29b (14). However, in the experimental model of Ang II-dependent hypertension, the expression of miR-133a was notably reduced under Ang II treatment, suggesting that high levels of Ang II are negatively related to miR-133a expression $(14,22)$.

To investigate the molecular mechanism of Ang II-induced hypertension, in this study, we employed miR-133a mimic and inhibitor to assess the inhibitory effect of Ang II on the levels of miR-133a. We also measured the expression of PRR under Ang II treatment and miR-133a mimic/inhibitor to explore the possible role of PRR in Ang II-dependent hypertension.

\section{Materials and methods}

Cell culture and identification. Human umbilical vein endothelial cells (HUVECs) were obtained from the Global Bioresource Center [American Type Culture Collection (ATCC) PCS-100-010 ${ }^{\mathrm{TM}}$ ]. The cells were maintained in Dulbecco's modified Eagle's medium (DMEM; ATCC $30-2002^{\mathrm{TM}}$ ) containing $10 \%$ fetal bovine serum (FBS; ATCC 30-2020 ${ }^{\mathrm{TM}}$ ) in a humidified atmosphere of $5 \% \mathrm{CO}_{2}$ at $37^{\circ} \mathrm{C}$. The medium was replaced every 2 days until the cells reached $\sim 80-90 \%$ confluency. After treatment with trypsin, the cells were transferred to multi-well plates for further culture.

After 1 week of culture, the HUVECs were identified using immunofluorescence staining. HUVECs were rinsed with $0.1 \mathrm{M}$ PBS. HUVECs were fixed in $100 \%$ methanol at $4^{\circ} \mathrm{C}$ for $30 \mathrm{~min}$ after attaining $90 \%$ confluence. Cells were treated with PBS containing $0.25 \%$ Triton X-100 (T8200; Beijing Solarbio Science \& Technology, Co., Ltd.) for $30 \mathrm{~min}$ at room temperature to increase cell permeability. Cells were blocked with $3 \%$ bovine serum albumin (cat. no. 37525; Thermo Fisher Scientific, Inc.) in PBS for $60 \mathrm{~min}$ at room temperature. The cells were then incubated with rabbit anti-Fac VIII antibody (1:400, ab6994; Abcam) overnight at $4^{\circ} \mathrm{C}$. After removing the primary antibody by rinsing with $\mathrm{PBS}$, the cells were cultured with secondary Goat anti rabbit IgG (Alexa Fluor ${ }^{\circledR} 488$, 1:400, ab150077; Abcam) at room temperature for $2 \mathrm{~h}$ in the dark. DAPI (Wuhan Boster Bio Engineering Co., Ltd.) was used to stain the cell nuclei (blue) at room temperature for $10 \mathrm{~min}$.
After washing with PBS, the cells were analyzed under a fluorescence microscope (Olympus Corporation, magnification, x400) and images were captured with a DP70 digital camera (Olympus Corporation), 5 areas of per section were captured. PBS was used in place of the antibodies in the control condition.

Survival of HUVECs with Ang II treatment. The effects of Ang II (IA0380, Beijing Solarbio Science \& Technology, Co., Ltd.) on HUVECs were examined using an MTT assay (cat. no. M2128, Sigma-Aldrich; Merck KGaA). HUVECs were seeded in 96-well plate, and adjusted to $4 \times 10^{3}$ cells/well with DMEM containing 10\% FBS. The cell suspension was divided into several groups for Ang II pretreatment of different concentrations $\left(0,10^{-7}, 10^{-6}, 10^{-5}\right.$ and $\left.10^{-4} \mathrm{M}\right)$; the control group remained untreated. As described previously (23), three time points were set $(24,48$ and $72 \mathrm{~h}$ ) to analyze the potential time-dependent effects of the Ang II on cell viability. Subsequently, the plates were incubated with MTT solution for $4 \mathrm{~h}$. The supernatant was removed, and DMSO (cat. no. D2650, Sigma-Aldrich; Merck KGaA) was added to dissolve formazan crystals. The absorbance was measured at $450 \mathrm{~nm}$ in a microplate reader.

Reverse transcription-quantitative polymerase chain reaction (RT-qPCR). To investigate the effect of Ang II with different concentrations on miR-133a expression, TRIzol reagent (Thermo Fisher Scientific, Inc.) was used to extract total miRNAs and mRNAs from Ang II-treated HUVECs. cDNA was synthesized from RNA using the PrimeScript ${ }^{\mathrm{TM}}$ 1st Strand cDNA Synthesis kit (Takara Bio, Inc.) with miRNA-specific primers (Table I). The $20 \mu 1$ reaction mixture was incubated under the following parameters: Firstly, $65^{\circ} \mathrm{C}$ for $5 \mathrm{~min}$, secondly, $30^{\circ} \mathrm{C}$ for $6 \mathrm{~min}$ and $50^{\circ} \mathrm{C}$ for $50 \mathrm{~min}$. The mRNA levels were amplified using the SYBR Premix Ex Taq $^{\mathrm{TM}}$ II in the 7500 Real-Time PCR system (Applied Biosystems; Thermo Fisher Scientific, Inc.). The $20 \mu \mathrm{l}$ qPCR reaction solution contained $1 \mu \mathrm{l}$ each of the forward and reverse primers $(10 \mu \mathrm{M}), 10 \mu \mathrm{l}$ SYBR fluorescent dye, $2 \mu \mathrm{l} \mathrm{cDNA}$ and RNase Free $\mathrm{dH}_{2} \mathrm{O}$. qPCR reactions were performed as follows: Initial denaturation at $95^{\circ} \mathrm{C}$ for $10 \mathrm{~min}$, repeated amplification by 40 cycles of $95^{\circ} \mathrm{C}$ for $13 \mathrm{sec}, 60^{\circ} \mathrm{C}$ for $30 \mathrm{sec}$ and $72^{\circ} \mathrm{C}$ for $2 \mathrm{~min}$ and finally $72^{\circ} \mathrm{C}$ for $10 \mathrm{~min}$. GAPDH was employed as an internal control. The relative levels of miR-133a were calculated with the $2^{-\Delta \Delta \mathrm{Cq}}$ method (24).

Transfection of miR-133a mimics and inhibitor. The miR-133a mimics/inhibitor (cat. nos. 4464066 and 4464084) were obtained from Thermo Fisher Scientific, Inc. HUVECs were incubated in 6-well plates at $50 \%$ confluence. miR-133a mimics, miR-133a inhibitor and negative miRNA control (cat. no. 4464061) were diluted into $250 \mu \mathrm{l}$ DMEM to a concentration of $50 \mathrm{nM}$ and $5 \mu$ l Lipofectamine ${ }^{\circledR} 2000$ (Invitrogen; Thermo Fisher Scientific, Inc.) at room temperature. The mixture of diluted miRNA and transfection reagents were dispensed into plates and incubated at $37^{\circ} \mathrm{C}$ for $6 \mathrm{~h}$ in a humidified atmosphere of $5 \% \mathrm{CO}_{2}$; the media was then replaced complete medium (DMEM with $10 \% \mathrm{FBS}$ ). The next day, cells were harvested and the efficacy of transfection with the miR-133a mimics and inhibitor were tested by RT-qPCR. 
Table I. Primers employed for reverse transcription-quantitative polymerase chain reaction.

\begin{tabular}{ll}
\hline Gene & \multicolumn{1}{c}{ Primer sequences } \\
\hline MicroRNA-133a & Forward: 5'-ATAAGAATGCGGCCGCATTCCAAACTAGCAGCACTA-3' \\
& Reverse: 5'-AGCTTTGTTTAAACTTAACCATTCTAGCTTTTCC-3' \\
Prorenin receptor & Forward: 5'-CAGACGTGGCTGCATTGTCC-3' \\
& Reverse: 5'-CTGGGGGTAGAGCCAGTTTGTT-3 \\
GAPDH & Forward: 5'-ATGGCACCGTCAAGGCTGAG-3' \\
& Reverse: 5'-TGTCAGGTACGGTAGTGACG-3 \\
\hline
\end{tabular}

An MTT assay was used to perform the cell viability of all groups' cells at 24,48 and $72 \mathrm{~h}$.

Effects of miR-133a mimics/inhibitor on the viability of Ang II-induced HUVECs. HUVECs transfected with miR-133a mimics/inhibitor and empty vector were incubated with Ang II and FBS-free DMEM to assess the effects of miR-133a mimics/inhibitor on Ang II $\left(10^{-5} \mathrm{M}\right)$-induced HUVECs. The viabilities of treated cells were compared with the control group (untreated cells), and cells transfected with miR-133a mimics/inhibitor and empty vector (without Ang II treatment) groups were determined via an MTT assay every $24 \mathrm{~h}$ as aforementioned.

Flow cytometry for the detection of apoptotic Ang II-induced HUVECs. Cells in all groups were diluted to $4 \times 10^{3}$ cells/well with complete medium in 96-well plates. After 48 h, HUVECs were trypsinized and harvested by centrifugation at $1,000 \mathrm{x} \mathrm{g}$ for $10 \mathrm{~min}$ at $4^{\circ} \mathrm{C}$. Cells were resuspended in binding buffer with propidium iodide (PI) and Annexin V-fluorescein isothiocyanate. The stained cells were incubated at dark place for $15 \mathrm{~min}$ at room temperature. Then, the apoptosis of various treated HUVECs was assessed immediately with a FACScalibur flow cytometer (BD Biosciences, San Jose, CA, USA) equipped with CellQuest software (version 3.3; BD Biosciences).

miR-133a target prediction and a dual luciferase assay. TargetScan (version 7.1, http://www.targetscan.org/vert_71/) was used to predict potential target genes of miR-133a and identified PRR (ATPase $\mathrm{H}+$ transporting accessory protein 2) as a potential target. Firstly, we cloned miR-133a into the GV272 vector (GV268-miR-133a; Shanghai GeneChem Co., Ltd.). In addition, we inserted the target sequences of miR-133a (accession no. NM_005765) present in the 3'untranslated region of PRR into the GV268 plasmid vector (GV272-PRR 3'UTR). Secondly, after mutation using the QuikChange Multi Site-Directed Mutagenesis kit (Agilent Technologies, Inc.) the hsa-miR-133a-3p.1 (miRbase, accession no. MIMAT0000427) sequences used were changed from 5'-UUGGUCCCCUUC AACCAGCUG-3' to 5'-UUCCACCCCUUCAACCAGCUG-3', which was predicted to abolish binding; the mutated fragment was inserted into GV268 plasmid vector (GV268-miR-133a-mut).

Luciferase reporter experiments were performed in a human kidney epithelial cell line (293T, ATCC CRL-3216 ${ }^{\mathrm{TM}}$; ATCC) using Dual-Luciferase ${ }^{\circledR}$ Reporter Assay System (Promega Corporation). 293T cells were cultured in an incubator with $5 \% \mathrm{CO}_{2}$ at $37^{\circ} \mathrm{C}$ and harvested at $80 \%$ confluence, and then seeded into a 24-well plate with complete medium. Cells were transfected with $50 \mathrm{ng}$ of GV272-PRR 3'UTR plasmid, $40 \mathrm{nM}$ of control or GV268-miR-133a or GV268-miR-133a-mut were transfected into cells using Lipofectamine 2000 as aforementioned. After $48 \mathrm{~h}$ post-transfection, $293 \mathrm{~T}$ cells were lysed and separated by centrifugation at $15,000 \mathrm{xg}$ for $3 \mathrm{~min}$ at $4^{\circ} \mathrm{C}$. The prepared dual luciferase reporter mixture was added into the supernatant of lysed cells and the relative luciferase activity was immediately measured by Sinergy 2 luminometer (Biotek Instruments, Inc.), Renilla luciferase activity was used for normalization.

$P R R$ expression in the miR-133a mimics/inhibitor transfected HUVECs. The expression levels of PRR in HUVECs transfected with miR-133a mimics, miR-133a inhibitor, or empty vector were measured by RT-qPCR as described above and western blotting. The primers of PRR were presented in Table I. A BCA protein assay was used to extract the total proteins from all cell groups. 10\% SDS-PAGE was used to separate proteins $(30 \mu \mathrm{g})$, and the membrane was transferred to a polyvinylidene difluoride membrane (EMD Millipore) and blocked with 5\% nonfat milk in TBST at room temperature for $60 \mathrm{~min}$. The membrane was incubated with rabbit anti-PRR (1:1,000, HPA003156; Sigma-Aldrich; Merck KGaA) primary antibodies at $4^{\circ} \mathrm{C}$ overnight. Subsequently, the membrane was washed three times in TBST for $10 \mathrm{~min}$ at room temperature, and then was incubated with the goat anti-rabbit IgG, HRP-linked antibody (1:10,000, cat. no. 31460; Thermo Fisher Scientific, Inc.) for $2 \mathrm{~h}$ at room temperature. Proteins were detected using $\mathrm{ECL}^{\mathrm{TM}}$ western blot detection reagents (cat. no. 32106; Thermo Fisher Scientific, Inc.). Optical band density was quantified by ImageJ software (version 1.46; National Institutes of Health). GAPDH was considered as an internal control.

Effects of PRR silencing on miR-133a inhibitor-transfected HUVECs under Ang II treatment. Small interfering RNA specifically targeting PRR (siPPR; cat. no. 138075) was obtained from Invitrogen (Thermo Fisher Scientific, Inc.). Lipofectamine 2000, the restriction enzymes BamHI (cat. no. ER0051) and EcoRI (cat. no. ER0271) (both from Thermo Fisher Scientific, Inc.) and the pcDNA3.1 (+) vector (Shanghai GenePharma Co., Ltd.) was used to transfect siPRR $(50 \mathrm{nM})$ into untreated cells and miR-133a inhibitor-treated cells (miR-133a inhibitor + siPRR), according to the manufacturer's protocols. Following transfection for $48 \mathrm{~h}$, cells were harvested and used for subsequently experiments. To explore the effects of siPRR, miR-133a inhibitor and Ang II treatment 
A

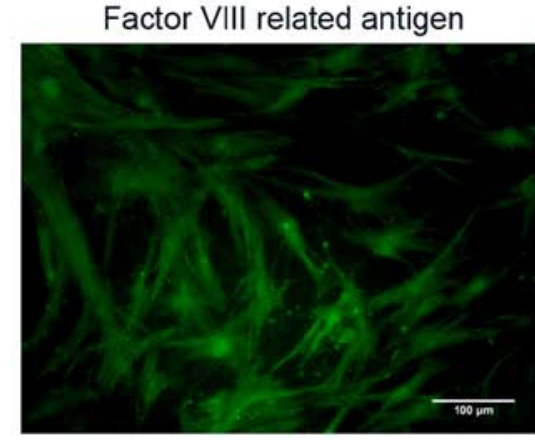

DAPI

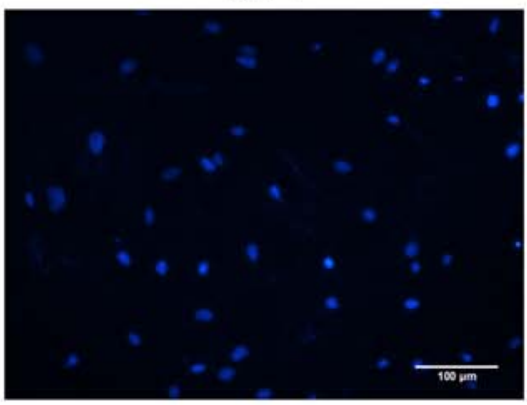

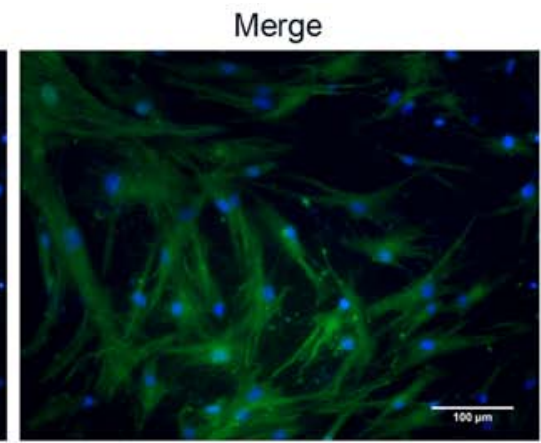

C

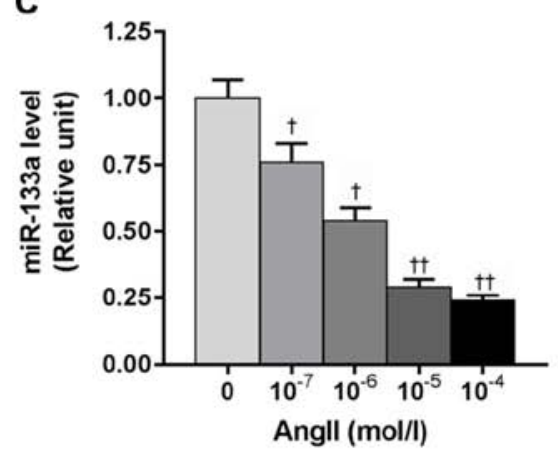

Figure 1. Immunofluorescence staining of HUVECs for von Willebrand factor. (A) Factor VIII-related antigen: Immunofluorescent staining of HUVECs with antibodies against von Willebrand factor (green). DAPI was used to stain the nuclei of HUVECs blue. Merge: HUVECs were co-stained with DAPI and von Willebrand factor antibody. Magnification, x100. (B) HUVECs were treated with different concentrations of Ang II. (C) The effects of different concentrations of AngII on the expression of miR-133a. Data are presented as the mean \pm standard error of the mean $(\mathrm{n}=3)$. GAPDH was used as an internal control. ${ }^{\dagger} \mathrm{P}<0.05$, ${ }^{+\dagger} \mathrm{P}<0.01$ vs. untreated HUVECs. Ang II, angiotensin II; HUVECs, human umbilical vein endothelial cells; miR, microRNA.

on PRR expression, seven groups were generated, including control, empty vector (NC), siPRR, NC + Ang II, miR-133a inhibitor + Ang II, NC + Ang II + siPRR and miR-133a inhibitor + Ang II + siPRR. The relative levels of PRR were determined by RT-qPCR and western blotting as described above. We also measured the rate of apoptosis of these cell groups by flow cytometry as described above, the advanced apoptotic cells in the upper right quadrant and early apoptotic cells in the lower right quadrant, the apoptotic rate was the sum of the advanced apoptotic rate and the early apoptotic.

Statistical analysis. Statistical significance was analyzed by using one-way analysis of variance between groups, followed by a Bonferroni's post-hoc test using SPSS version 20.0 (IBM Corp.). All data analysis were performed three times. All data were presented as the mean \pm standard error of the mean. $\mathrm{P}<0.05$ was considered to indicate a statistically significant difference.

\section{Results}

Cultured cells are identified as HUVECs by immunofluorescence analysis. As shown in Fig. 1, the notable green and blue fluorescence indicated positive identification of factor VIII-related antigen. Cell nuclei were stained with DAPI, in which the blue fluorescence was evenly distributed in the nucleus. Our results demonstrated the obtained cells were highly purified HUVECs.

Ang II suppresses cell viability and miR-133a expression in HUVECs. Our results showed that Ang II inhibited the viability of HUVECs in a time- and concentration-dependent manner, while the expression of miR-133a was reduced with increasing concentrations of Ang II. In addition, Ang II of $10^{-5} \mathrm{M}$ significantly suppressed the viability of HUVECs at $48 \mathrm{~h}$ compared with the control; a significant decrease in miR-133a expression was also noted (Fig. 1B and C). Therefore, Ang II of $10^{-5} \mathrm{M}$ was selected for subsequent experiments.

Downregulated miR-133a suppresses the viability of HUVECs. To investigate the effects of miR-133a on the viability of HUVECs, cells were transfected with miR-133a mimics or inhibitors (Fig. 2A). Our results revealed that overexpression of miR-133a markedly enhanced the viability of HUVECs, whereas miR-133a inhibitors exerted opposing effects on HUVECs and significantly reduced the cell viability at $72 \mathrm{~h}$, compared with the control and miR-133a mimics groups (Fig. 2B).

Upregulated miR-133a enhances the viability of Ang II-induced HUVECs. Our results showed the cell viabilities in the miR-133a inhibitor + Ang II and NC + Ang II groups to be significantly decreased at $48 \mathrm{~h}$, compared with the control and NC groups. miR-133a mimic had a notably positive effect on cell viability. The cell viability in miR-133a inhibitor group was significantly reduced at $72 \mathrm{~h}$ compared with the control; the cell viability in miR-133a inhibitor + Ang II group was the lowest (Fig. 3A).

Flow cytometry was used to determine the apoptotic rates of the various treated HUVECs (Fig. 3B). The apoptotic rate of miR-133a inhibitor-transfected cells significantly increased from 

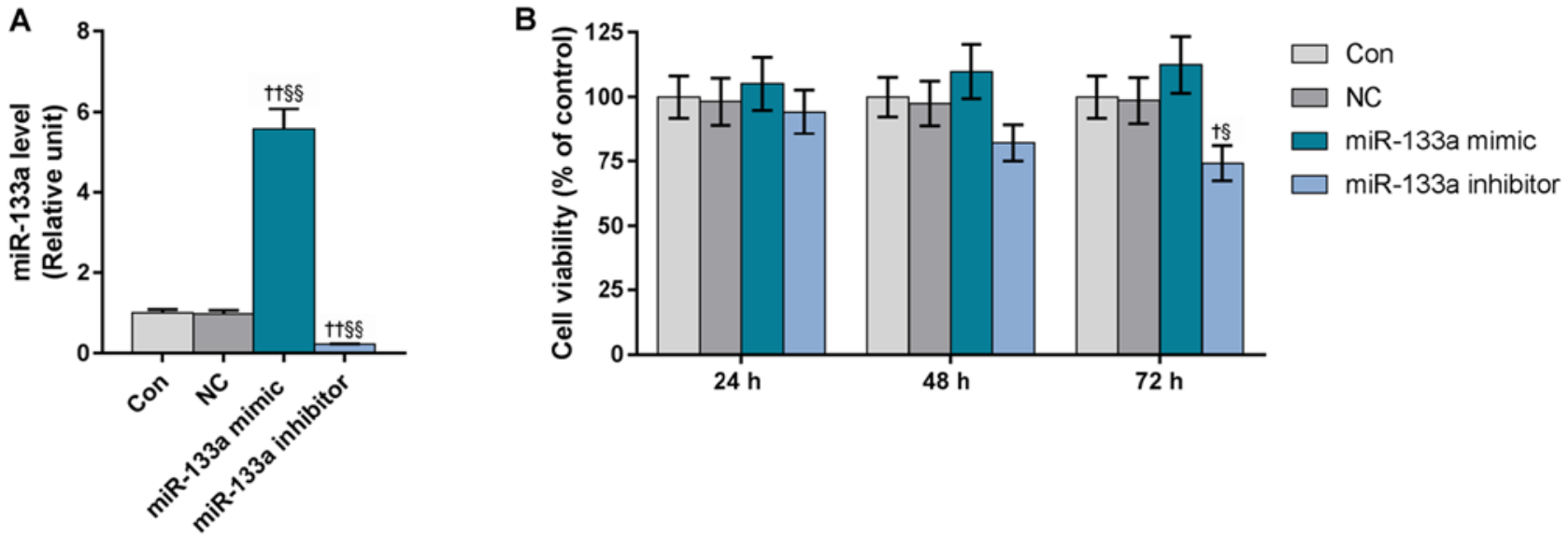

Figure 2. Downregulated miR-133a has a negative effect on the viability of HUVECs. (A) The levels of miR-133a were detected in HUVECs transfected with miR-133a mimic or inhibitor by reverse transcription-quantitative polymerase chain reaction. (B) The cell viability of all groups was measured every $24 \mathrm{~h}$ via an MTT assay. Data are presented as the mean \pm standard error of the mean $(n=3)$. GAPDH was used as an internal control. ${ }^{+} \mathrm{P}<0.05,{ }^{\dagger+} \mathrm{P}<0.01 \mathrm{vs}$. control group; ${ }^{\S} \mathrm{P}<0.05,{ }^{\S} \mathrm{P}<0.01$ vs. NC group. Con, control; miR, microRNA; NC, negative control.
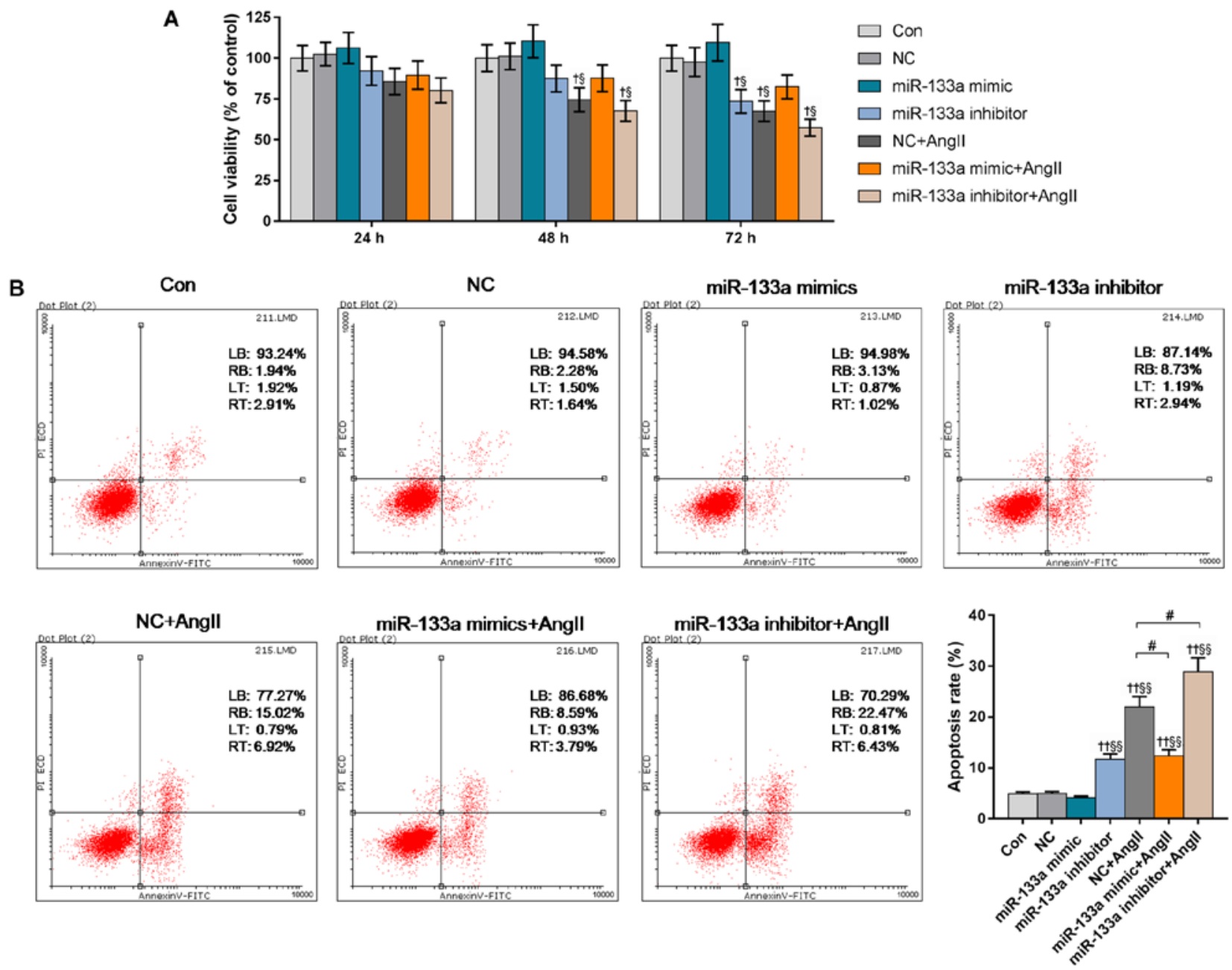

Figure 3. Expression levels of miR-133a are positively associated with the viability and apoptosis of HUVECs. (A) Cell viability was examined in the HUVECs transfected with miR-133a mimic/inhibitor under treatment with $10^{-5} \mathrm{M}$ Ang II. (B) The apoptotic rates of the cell groups were determined via flow cytometry. Data were presented as the mean \pm standard error of the mean $(\mathrm{n}=3)$. GAPDH was used as an internal control. ${ }^{+} \mathrm{P}<0.05,{ }^{+\dagger} \mathrm{P}<0.01 \mathrm{vs}$. control group; ${ }^{\circledR} \mathrm{P}<0.05,{ }^{\$ \S} \mathrm{P}<0.01$ vs. NC group; ${ }^{\#} \mathrm{P}<0.05$ vs. NC + Ang II group. Ang II, angiotensin II; HUVECs, human umbilical vein endothelial cells; miR, microRNA; $\mathrm{NC}$, negative control.

$4.85 \%$ in the control to $11.67 \%(\mathrm{P}<0.01)$. miR-133a mimic markedly reduced the Ang II-induced apoptotic rate from $21.94 \%$ in the $\mathrm{NC}+$ Ang II group to $12.38 \%(\mathrm{P}<0.05)$. The miR-133a inhibitor + Ang II group had the highest apoptotic rate (28.9\%). 
A

\begin{tabular}{|c|c|}
\hline $\begin{array}{l}\text { Position 834-840 of ATP6AP2 3' UTR } \\
\text { hsa-miR-133a-3p.1 } \\
\text { hsa-miR-133a-3p.1 mut }\end{array}$ & $\begin{array}{l}5 \text {...UUUUUAUGUUGGAGUGGACCAAU.. } \\
\text { I I I I I I II II } \\
3 \times \text { GUCGACCAACUUCCCCUGGUU } \\
3 \times \text { GUCGACCAACUUCCCCACCUU }\end{array}$ \\
\hline
\end{tabular}

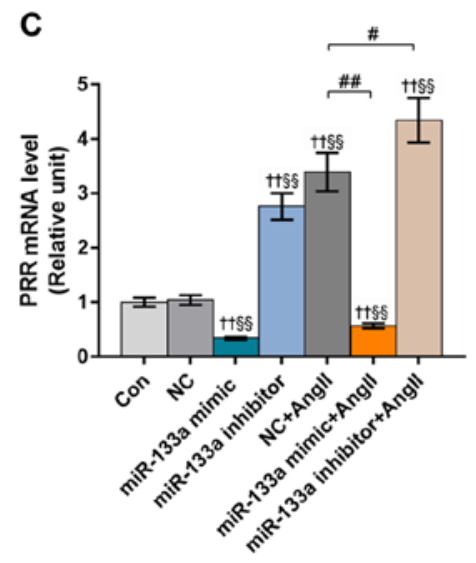

D

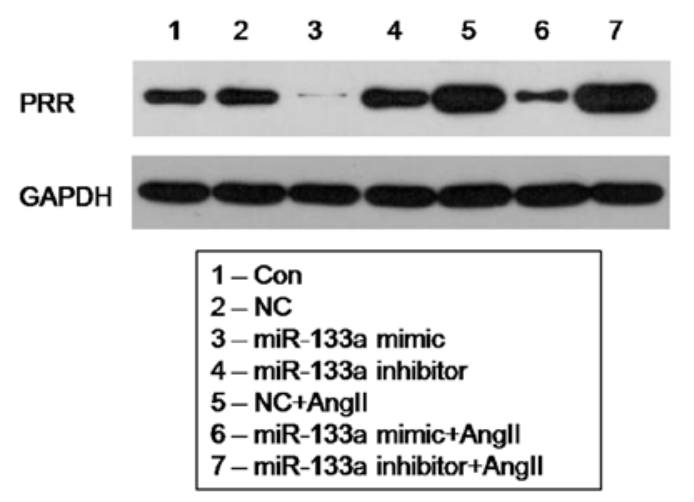

B

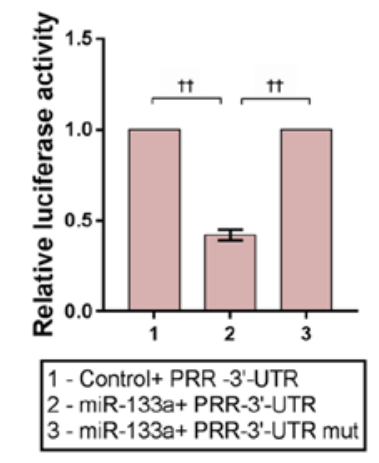

Figure 4. PRR is a direct target of and is mediated by miR-133a. (A) The binding sites of PRR and miR-133a, and the induced mutation of miR-133a. (B) PRR was targeted by miR-133a, but not the miR-133a mut. (C and D) miR-133a expression was negatively related to that of PRR as detected by reverse transcription-quantitative polymerase chain reaction and western blotting. Data were presented as the mean \pm standard error of the mean $(n=3)$. GAPDH was detected as an internal control. ${ }^{+\dagger} \mathrm{P}<0.01$ vs. control group or as indicated; ${ }^{\circledR \S} \mathrm{P}<0.01$ vs. $\mathrm{NC}$ group; ${ }^{\#} \mathrm{P}<0.05,{ }^{\# \#} \mathrm{P}<0.01$ vs. NC + Ang II group. Ang II, angiotensin II; miR, microRNA; mut, mutated; NC, negative control; PRR, prorenin receptor; UTR, untranslated region.

miR-133a targets the 3'UTR of PRR $m R N A$. In the first step of target prediction, we used TargetScan and identified two potential binding sites for miR-133a in the position 834-840 of human PRR 3'UTR. Bioinformatic analysis for the target site of miR-133a in the 3'-UTR of PRR was shown in Fig. 4A. We also performed a dual-luciferase reporter assay to further verify the putative direct binding of miR-133a to the 3'UTR of PRR mRNA.

$293 \mathrm{~T}$ cells were co-transfected with plasmids containing the 3'-UTR of PRR (GV272-PRR 3'UT), and miR-133a (GV268-miR-133a) or miR-133a-mut plasmid (GV268-miR-133a-mut). The results of the luciferase assay revealed that the activity of firefly luciferase in the miR-133a-transfected group was significantly reduced compared with the control, while no significant changes were observed in miR-133a-mut transfected group (Fig. 4B).

We further examined the expression levels of PRR in the miR-133a mimic/inhibitor-transfected HUVECs. The results of RT-qPCR and western blotting demonstrated that the levels of PRR in miR-133a mimic-transfected HUVECs were significantly lower than the control groups, while the mRNA level of PRR in miR-133a inhibitor group and NC + Ang II group were significantly higher compared with the control groups; and miR-133a inhibitor significantly enhanced the promotive effect of Ang II on PRR expression (Fig. 4C and D). These results indicated that Ang II promotes PRR expression by inhibiting miR-133a.

siPRR weakens the proapoptotic effects of Ang II and miR-133a inhibitor on HUVECs. We employed siPRR to investigate the molecular mechanism of Ang II acting on PRR expression. Our results showed that Ang II induced significantly increased the expression of PRR, compared with the control and NC groups $(\mathrm{P}<0.01)$; however, the miR-133a inhibitor + Ang II group had a significantly higher expression of PRR than the NC + Ang II group $(\mathrm{P}<0.05)$. After being transfected with siPRR, the PRR levels were significantly decreased compared with the control; the relative expression levels of PRR in the miR-133a inhibitor + Ang II + siPRR group was significantly higher than in the $\mathrm{NC}+$ Ang II + siPRR group $(\mathrm{P}<0.05)($ Fig. 5A and B).

We further measured the apoptosis of all cell groups by flow cytometry. The apoptotic rate of the siPRR group exhibited no significant difference compared with the control and NC groups, but Ang II treatment significantly enhanced apoptosis from $4.77 \%$ in the control to $21.49 \%$. miR-133a inhibitor significantly enhanced the apoptosis-promoting effects of Ang II $(\mathrm{P}<0.05)$. Importantly, siPRR significantly reduced the apoptotic rate from $21.49 \%$ in the NC + Ang II group and $28.52 \%$ in the miR-133a inhibitor + Ang II group to 11.39 and $12.94 \%$, respectively $(\mathrm{P}<0.01)$ (Fig. 5C).

\section{Discussion}

Endothelial cells play a crucial role in regulating the production of Ang and vasopermeability $(25,26)$. Endothelial dysfunction was considered as an initiation factor in the pathophysiology of essential hypertension and subsequently induced various complications (27). Previous studies have reported that miRNAs were extensively involved in the occurrence of 
A

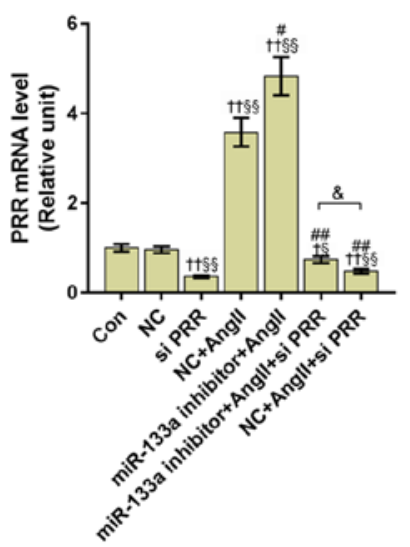

B

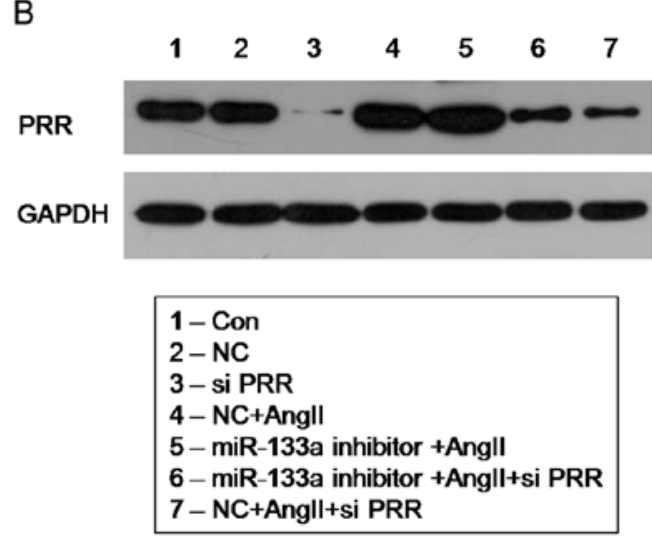

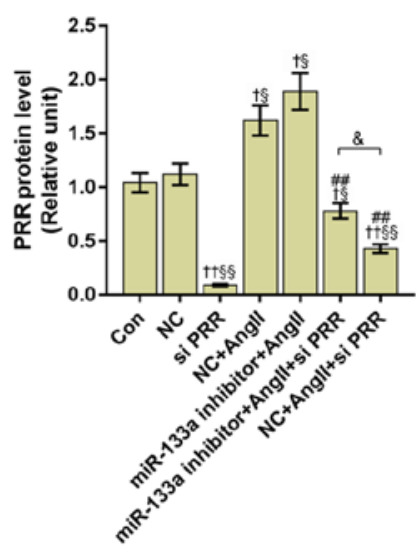

C

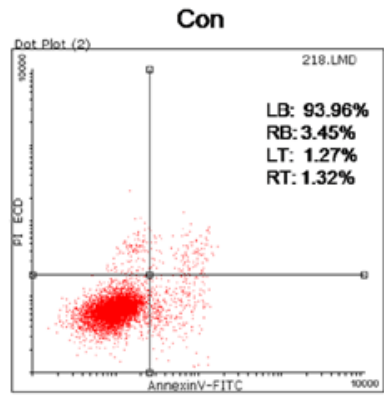

miR-133a inhibitor+Angll

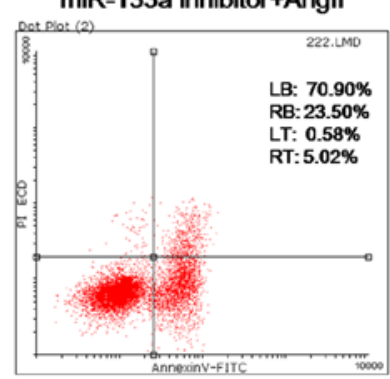

NC

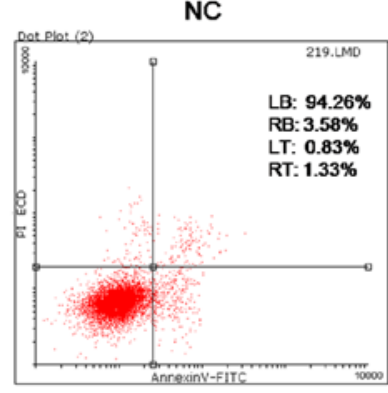

miR-133a inhibitor+Angll+si PRR

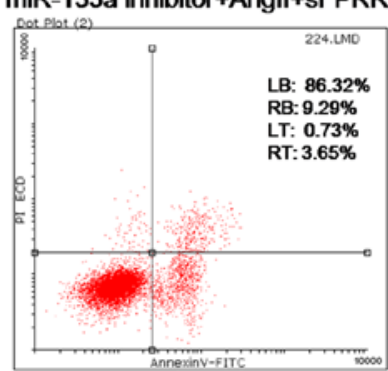

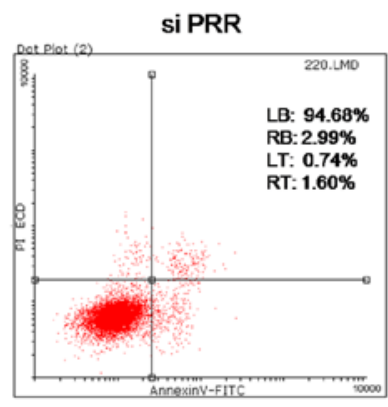
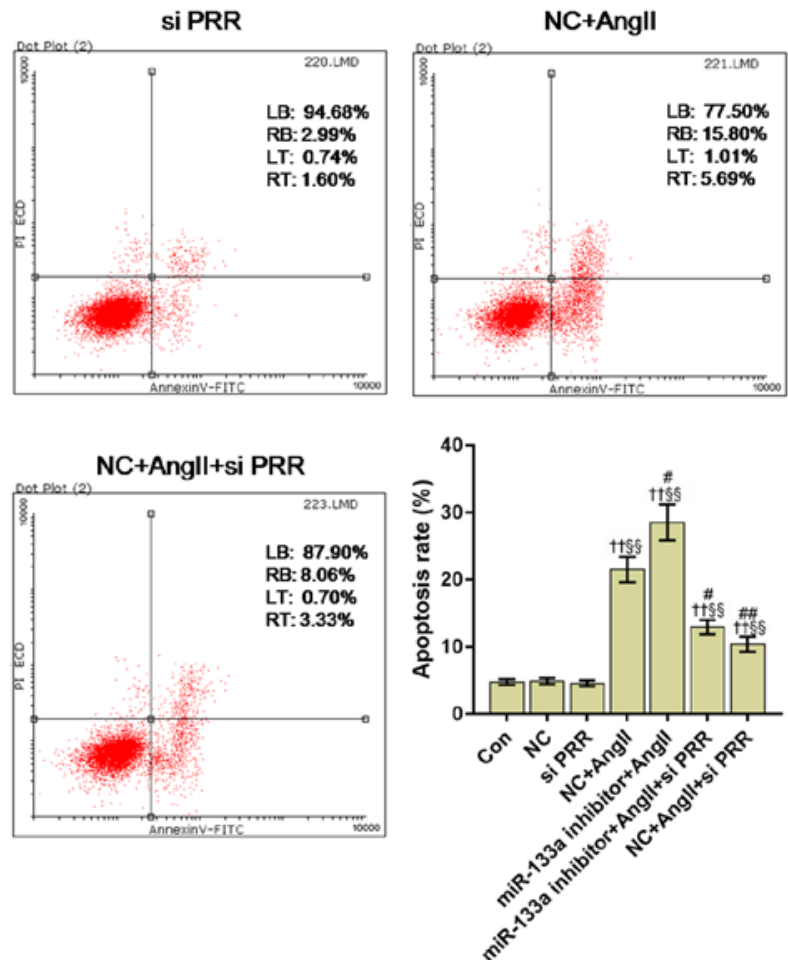

Figure 5. siPRR suppresses the pro-apoptotic ability of Ang II and miR-133a inhibitor. (A and B) The effects of siPRR on the levels of PRR expression in HUVECs treated with Ang II and miR133a inhibitor as measured by reverse transcription-quantitative polymerase chain reaction and western blotting. (C) Apoptosis of all HUVEC groups as determined by flow cytometry. Data are presented as the mean \pm standard error of the mean ( $\mathrm{n}=3$ ). GAPDH was detected as an internal control. ${ }^{+} \mathrm{P}<0.05,{ }^{++} \mathrm{P}<0.01$ vs. control group; ${ }^{\S} \mathrm{P}<0.05,{ }^{\S} \mathrm{P}<0.01$ vs. NC group; ${ }^{\#} \mathrm{P}<0.05,{ }^{\# \#} \mathrm{P}<0.01$ vs. NC + Ang II group; ${ }^{\text {\& }} \mathrm{P}<0.05$ vs. NC + Ang II + siPRR. Ang II, angiotensin II; miR, microRNA; NC, negative control; PRR, prorenin receptor.

hypertension, and served an important role in the regulation of blood pressure $(28,29)$. In present study, we observed that miR-133a had the ability to directly modulate the expression of PRR at the transcriptional level; Ang II was proposed to significantly upregulate PRR expression to enhance the apoptosis of HUVECs through the dysregulation of miR-133a levels.

Numerous reports have suggested that Ang II, as a major effector peptide of the RAS, was closely related to the occurrence and development of hypertension $(30,31)$. Our results showed that Ang II treatment could significantly suppress the viability of HUVECs with increasing concentrations of Ang II, which may promote the process of endothelial dysfunction and hypertension. The effects of Ang II on HUVEC viability also occurred in a time-dependent manner. Several previous studies showed that the endogenous miRNAs produced by endothelial cells regulated the expression of hypertension-associated genes $(18,32,33)$. In 2012, Castoldi et al (14) revealed that downregulated miR-133a was detected in cardiac hypertrophy in transgenic mice with cardiac overexpression of an active mutant protein kinase B; Kontaraki et al (20) demonstrated that miR-133a was negatively associated with left ventricular hypertrophy. These findings were consistent with our results, in which the expression of miR-133a was significantly inhibited in Ang II-induced HUVECs at the concentration of $10^{-5} \mathrm{M}$. To explore the relationship between miR-133a expression and the suppressed viability of HUVECs induced by Ang II, miR-133a mimics and inhibitor were employed. We reported that miR-133a mimics counteracted the inhibitory effects of Ang II on miR-133a expression, but miR-133a inhibitor 
could promote the apoptosis induced by Ang II treatment. These results suggested that Ang II enhanced the apoptosis of HUVECs through the dysregulation of miR-133a expression, while increased miR-133a levels may suppress apoptosis.

Recently, PRR was reported in the molecular mechanism of hypertension as a member of RAS $(34,35)$. Several researches detected upregulated PRR in Ang II-dependent hypertension $(11,36)$, which was consistent with our experiments whereby the expression levels of PRR were significantly increased following Ang II treatment. In 2015, Li et al (10) designed and developed a novel PRR inhibitory peptide (PRO20), which could efficiently inhibit PRR binding to prorenin and Ang II-dependent hypertension. Of note, miR-133a inhibitor was observed to induce the expression of PRR, while, miR-133a mimics suppressed PRR expression with or without Ang II treatment. It has been known that miRNAs typically bind to the mRNA 3'-UTR of its target gene or lead to the degradation the mRNA at the post-transcriptional level, thereby inhibiting the expression of the target gene (37-39). A previous study found that miR-152 could modulate the RAS through directly targeting PRR under hyperglycemic conditions (40). In 2018, Wang etal (36) reported that 30 differentially expressed miRNAs were predicted to target RAS components, and transfection of miR-181a-5p and miR-663 into HTR-8/SVneo trophoblast cells suppressed the mRNA expression of genes encoding prorenin, and prorenin protein production (41). However, no sufficient role of miR-133a in the PRR-protein-angiotensin system was determined. Therefore, we evaluated the targeting ability of miR-133a to PRR mRNA by using TargetScan and conducting a dual-luciferase assay, and confirmed that PRR was a target of miR-133a. The PRR 3' UTR was determined to contain two predicted conserved target sites for miR-133a.

In present study, siPRR was successfully transfected into HUVECs to investigate the role of PRR in cell apoptosis mediated by Ang II. Our results demonstrated that siPRR had no notable effect on cell apoptosis compared with the control and $\mathrm{NC}$ groups, yet the apoptosis of the NC + Ang II and miR-133a inhibitor + Ang II groups were significantly decreased by PRR silencing. Taken together, our results suggested that siPRR could significantly suppress the proapoptotic ability of Ang II and miR-133a inhibitor.

There are some limitations to our study. The observation that miR-133a downregulation promoted HUVECs injury was only supported by in vitro experiments only. The effects of miR 133 on myocardial fibrosis in vivo and in vitro were also not evaluated, which poses as a limitation. Additionally, the mechanism we proposed requires further investigation.

In conclusion, we investigated the roles of Ang II, miR-133a and PRR in the molecular mechanism of hypertension. We reported that Ang II may serve in a negative feedback mechanism in association with miR-133a in the context of HUVEC viability. Ang II had the ability to gradually reduce cell viability of HUVECs with increasing concentrations, but this could be counteracted with increased miR-133a expression. miR-133a was demonstrated to be able to directly bind the PRR 3'UTR and inhibit PRR expression at the post-transcriptional level. Most importantly, our results suggested a possible mechanism in which Ang II inhibited the expression of miR-133a, while increased PRR promoted the apoptosis of HUVECs in the absence of miR-133a downregulation. These findings may improve understanding of the mechanism underlying Ang II-dependent hypertension and could aid developments into treatments for this condition.

\section{Acknowledgements}

Not applicable.

\section{Funding}

No funding was received.

\section{Availability of data and materials}

The analyzed datasets generated during the study are available from the corresponding author on reasonable request.

\section{Authors' contributions}

BL and ML made substantial contributions to conception and design of the present study. HW, DZ, JL and JT performed data acquisition, data analysis and interpretation. BL and ML drafted the article/critically revised it for important intellectual content. All authors approved the final version to be published. All authors agree to be accountable for all aspects of the work in ensuring that questions related to the accuracy or integrity of the work are appropriately investigated and resolved.

\section{Ethics approval and consent to participate}

Not applicable.

\section{Patient consent for publication}

Not applicable.

\section{Competing interests}

The authors declare that they have no competing interests.

\section{References}

1. Hodgson TA and Cai L: Medical care expenditures for hypertension, its complications, and its comorbidities. Medical Care 39: 599-615, 2001.

2. Ponticos $M$ and Smith BD: Extracellular matrix synthesis in vascular disease: Hypertension, and atherosclerosis. J Biomed Res 28: 25-39, 2014.

3. Rizzoni D and Agabiti-Rosei E: Structural abnormalities of small resistance arteries in essential hypertension. Intern Emerg Med 7: 205-212, 2012.

4. Tang NP, Li H, Qiu YL, Zhou GM, Wang Y, Ma J and Mei QB: The effects of microgravity on blood vessels and vascular endothelial cells. Sheng Li Ke Xue Jin Zhan 45: 385-390, 2014 (In Chinese).

5. Bali A and Jaggi AS: Angiotensin II-triggered kinase signaling cascade in the central nervous system. Rev Neurosci 27: 301-315, 2016.

6. Zimmerman MC, Lazartigues E, Lang JA, Sinnayah P, Ahmad IM, Spitz DR and Davisson RL: Superoxide mediates the actions of angiotensin II in the central nervous system. Circ Res 91: 1038-1045, 2002.

7. Becari C, Oliveira EB and Salgado MC: Alternative pathways for angiotensin II generation in the cardiovascular system. Braz J Med Biol Res 44: 914-919, 2011. 
8. Naik P, Murumkar P, Giridhar R and Yadav MR: Angiotensin II receptor type 1 (AT1) selective nonpeptidic antagonists-a perspective. Bioorg Med Chem 18: 8418-8456, 2010.

9. Messerli FH and Bangalore S: Angiotensin receptor blockers reduce cardiovascular events, including the risk of myocardial infarction. Circulation 135: 2085-2087, 2017.

10. Li W, Sullivan MN, Zhang S, Worker CJ, Xiong Z, Speth RC and Feng Y: Intracerebroventricular infusion of the (Pro)renin receptor antagonist PRO20 attenuates deoxycorticosterone acetate-salt-induced hypertension. Hypertension 65: 352-361, 2015

11. Li XC and Zhuo JL: Recent updates on the proximal tubule Renin-angiotensin system in angiotensin II-Dependent hypertension. Curr Hypertens Rep 18: 63, 2016.

12. Gonzalez AA, Lara LS, Luffman C, Seth DM and Prieto MC: Soluble form of the (pro)renin receptor is augmented in the collecting duct and urine of chronic angiotensin II-dependent hypertensive rats. Hypertension 57: 859-864, 2011.

13. Morimoto S, Ando T, Niiyama M, Seki Y, Yoshida N, Watanabe D, Kawakami-Mori F, Kobori H, Nishiyama A and Ichihara A: Serum soluble (pro)renin receptor levels in patients with essential hypertension. Hypertens Res 37: 642-648, 2014.

14. Castoldi G, Di Gioia CR, Bombardi C, Catalucci D, Corradi B, Gualazzi MG, Leopizzi M, Mancini M, Zerbini G, Condorelli G, et al: MiR-133a regulates collagen 1A1: Potential role of miR-133a in myocardial fibrosis in angiotensin II-dependent hypertension. J Cell Physiol 227: 850-856, 2012.

15. Li W, Peng H, Mehaffey EP, Kimball CD, Grobe JL, van Gool JM, Sullivan MN, Earley S, Danser AH, Ichihara A and Feng Y: Neuron-specific (pro)renin receptor knockout prevents the development of salt-sensitive hypertension. Hypertension 63: 316-323, 2014

16. Gonzalez AA and Prieto MC: Roles of collecting duct renin and (pro)renin receptor in hypertension: Mini review. Ther Adv Cardiovasc Dis 9: 191-200, 2015.

17. Bartel DP: MicroRNA target recognition and regulatory functions. Cell 136: 215-233, 2009.

18. Kriegel AJ, Baker MA, Liu Y, Liu P, Cowley AW Jr and Liang M Endogenous microRNAs in human microvascular endothelial cells regulate mRNAs encoded by hypertension-related genes. Hypertension 66: 793-799, 2015.

19. Dorn GW II: MicroRNAs in cardiac disease. Transl Res 157: 226-235, 2011.

20. Kontaraki JE, Marketou ME, Parthenakis FI, Maragkoudakis S, Zacharis EA, Petousis S, Kochiadakis GE and Vardas PE: Hypertrophic and antihypertrophic microRNA levels in peripheral blood mononuclear cells and their relationship to left ventricular hypertrophy in patients with essential hypertension. J Am Soc Hypertens 9: 802-810, 2015.

21. Matkovich SJ, Wang W, Tu Y, Eschenbacher WH, Dorn LE, Condorelli G, Diwan A, Nerbonne JM and Dorn GW II: MicroRNA-133a protects against myocardial fibrosis and modulates electrical repolarization without affecting hypertrophy in pressure-overloaded adult hearts. Circ Res 106: 166-175, 2010.

22. Wu Y, Huang A, Li T, Su X, Ding H, Li H, Qin X, Hou L, Zhao Q, Ge X, et al: MiR-152 reduces human umbilical vein endothelial cell proliferation and migration by targeting ADAM17. FEBS Lett 588: 2063-2069, 2014

23. Han G, Wei Z, Cui H, Zhang W, Wei X, Lu Z and Bai X: NUSAP1 gene silencing inhibits cell proliferation, migration and invasion through inhibiting DNMT1 gene expression in human colorectal cancer. Exp Cell Res 367: 216-221, 2018.

24. Livak KJ and Schmittgen TD: Analysis of relative gene expression data using real-time quantitative PCR and the 2(-Delta Delta C(T)) method. Methods 25: 402-408, 2001.

25. Shan H,Zhang S, Wei X, Li X, Qi H, He Y, Liu A, Luo D and Yu X: Protection of endothelial cells against Ang II-induced impairment: Involvement of both PPARa and PPAR $\gamma$ viaPI3K/Akt pathway.Clin Exp Hypertens 38: 571-577, 2016.
26. Pourgholami MH, Khachigian LM, Fahmy RG, Badar S, Wang L, Chu SW and Morris DL: Albendazole inhibits endothelial cell migration, tube formation, vasopermeability, VEGF receptor-2 expression and suppresses retinal neovascularization in ROP model of angiogenesis. Biochem Biophys Res Commun 397 729-734, 2010.

27. Gkaliagkousi E, Gavriilaki E, Triantafyllou A and Douma S Clinical Significance of endothelial dysfunction in essential hypertension. Curr Hypertens Rep 17: 85, 2015.

28. Poliseno L, Tuccoli A, Mariani L, Evangelista M, Citti L, Woods K, Mercatanti A, Hammond S and Rainaldi G: MicroRNAs modulate the angiogenic properties of HUVECs. Blood 108: 3068-3071, 2006.

29. Ooi JY, Bernardo BC and Mcmullen JR: The therapeutic potential of miRNAs regulated in settings of physiological cardiac hypertrophy. Future Med Chem 6: 205-222, 2014.

30. Biancardi VC, Bomfim GF, Reis WL, Al-Gassimi S and Nunes KP: The interplay between Angiotensin II, TLR4 and hypertension. Pharmacol Res 120: 88-96, 2017.

31. Cha SA, Park BM and Kim SH: Angiotensin-(1-9) ameliorates pulmonary arterial hypertension via angiotensin type II receptor. Korean J Physiol Pharmacol 22: 447-456, 2018.

32. Condorelli G, Latronico MV and Cavarretta E: microRNAs in cardiovascular diseases: Current knowledge and the road ahead. J Am Coll Cardiol 63: 2177-2187, 2014.

33. Dluzen DF, Kim Y, Bastian P, Zhang Y, Lehrmann E, Becker KG, Noren Hooten $\mathrm{N}$ and Evans MK: MicroRNAs modulate oxidative stress in hypertension through PARP-1 regulation. Oxid Med Cell Longev 2017: 3984280, 2017.

34. Yang T: Crosstalk between (Pro)renin receptor and COX-2 in the renal medulla during angiotensin II-induced hypertension. Curr Opin Pharmacol 21: 89-94, 2015.

35. Williamson CR, Khurana S, Nguyen P, Byrne CJ and Tai TC: Comparative analysis of renin-angiotensin system (RAS)-related gene expression between hypertensive and normotensive rats. Med Sci Monit Basic Res 23: 20-24, 2017.

36. Wang L, Zhu Q, Lu A, Liu X, Zhang L, Xu C, Liu X, Li H and Yang T: Sodium butyrate suppresses angiotensin II-induced hypertension by inhibition of renal (pro)renin receptor and intrarenal renin-angiotensin system. J Hypertens 35: 1899-1908, 2017

37. Christodoulatos GS and Dalamaga M: Micro-RNAs as clinical biomarkers and therapeutic targets in breast cancer: Quo vadis? World J Clin Oncol 5: 71-81, 2014.

38. Lauressergues D, Couzigou JM, Clemente HS, Martinez Y, Dunand C, Bécard G and Combier JP: Primary transcripts of microRNAs encode regulatory peptides. Nature 520: 90-93, 2015.

39. Sharma NM, Nandi SS, Zheng H, Mishra PK and Patel KP: A novel role for miR-133a in centrally mediated activation of the renin-angiotensin system in congestive heart failure. Am J Physiol Heart Circ Physiol 312: H968-H979, 2017.

40. Haque R, Hur EH, Farrell AN, Iuvone PM and Howell JC: MicroRNA-152 represses VEGF and TGF $\beta 1$ expressions through post-transcriptional inhibition of (Pro)renin receptor in human retinal endothelial cells. Mol Vis 21: 224-235, 2015.

41. Wang Y, Lumbers ER, Arthurs AL, Corbisier de Meaultsart C, Mathe A, Avery-Kiejda KA, Roberts CT, Pipkin FB, Marques FZ, et al: Regulation of the human placental (pro)renin receptor-prorenin-angiotensin system by microRNAs. Mol Hum Reprod 24: 453-464, 2018.

This work is licensed under a Creative Commons Attribution-NonCommercial-NoDerivatives 4.0 International (CC BY-NC-ND 4.0) License. 